\title{
OFF-BROADSIDE MAIN BEAM DESIGN FOR FREQUENCY INVARIANT BEAMFORMERS
}

Wei Liu

\author{
Communications Research Group
}

Dept. of Electronic \& Electrical Engineering

University of Sheffield, U.K.

w.liu@shef.ac.uk
Stephan Weiss
Keywords: frequency invariant beamforming, broadband arrays, off-broadside main beam

\begin{abstract}
In a previously proposed design method for frequency invariant beamforming, the design for the case of an off-broadside main beam is not satisfactory. After a detailed analysis, we propose two methods to overcome this problem: one is to increase the length of the FIR filter attached to each sensor, as a result, we need to sample the transformed desired response more densely in the associated direction; the other one is to design a broadside main beam first, then it is convolved with appropriate steering delay filters. Design examples show that the two methods can provide satisfactory results.
\end{abstract}

\section{INTRODUCTION}

Recently, the design of beamformers with a frequency invariant response has attracted the interest of many researchers. One of the suggested methods is to optimize the array parameters with respect to the desired response using available convex optimization methods [1, 2, 3]. However, for large arrays, the number of coefficients to be optimized is extremely large. A systematic method was proposed in [4], which can be applied to one-dimensional (1-D), two-dimensional (2-D) and three-dimensional (3-D) arrays, where each array element is followed by its own primary filter and the outputs of these primary filters share a common secondary filter to form the final output. However, beyond the 1-D array case this design method can be very complicated.

Most recently, a new class of frequency invariant arrays exploiting the Fourier transform relationship between the array's spatial and temporal parameters and its beam pattern

\author{
Institute of Comms \& Signal Processing \\ Dept. of Electronic \& Electrical Engineering \\ University of Strathclyde, U.K. \\ stephan.weiss@eee.strath.ac.uk
}

was proposed [5, 6], where the design can be achieved based on a simple multi-dimensional inverse Fourier transforms. However, it has been found that with the same number of array sensors and array coefficients, the off-broadside main beam design is not as good as the broadside main beam in terms of its frequency invariance property.

In this paper we propose two methods to enhance the offbroadside main beam design. Firstly, we show that an increase of the temporal dimension of the beamformer in combination with a denser sampling grid in the Fourier domain can achieve the desired result. Secondly, we design a broadside main beam in the first step and then achieve an off-broadside response by time-domain convolution with a series of FIR filters with appropriate fractional delays [7].

This paper is organised as follows. In Section 2 a review of the FIB design for linear arrays will be provided, then the problem for off-broadside main beam design will be highlighted. The two solutions will be proposed with corresponding design examples in Section 3 and arising problems will also be discussed. Conclusions are drawn in Section 4.

\section{FREQUENCY INVARIANT BEAMFORMING}

\subsection{Design for a uniformly spaced linear array}

An equally spaced linear array with a sensor spacing of $d_{x}$ is shown in Fig. 1. The received signal by the $m$-th sensor is sampled with a sampling period of $T$ and then processed by an FIR filter with coefficients $d[m, k], m=0, \ldots, M-1$, $k=0, \ldots, K-1$. Then the array's response can be written as

$$
R(\Omega, \theta)=\sum_{m=0}^{M-1} \sum_{k=0}^{K-1} d[m, k] \cdot e^{-j m \mu \Omega \sin \theta} \cdot e^{-j k \Omega},
$$




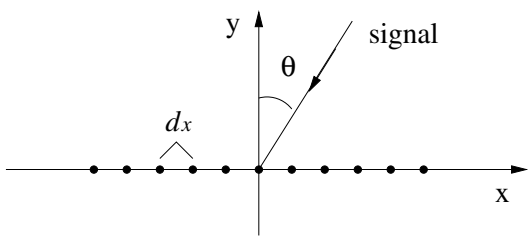

Fig. 1. A uniformed spaced linear array.

where $\mu=\frac{d_{x}}{c T}, \Omega=\omega T$ is the normalised angular frequency and $c$ the wave propagation speed. With the substitutions $\Omega_{1}=\mu \Omega \sin \theta$ and $\Omega_{2}=\Omega$ in (1), we have

$$
P\left(\Omega_{1}, \Omega_{2}\right)=\sum_{m=0}^{M-1} \sum_{k=0}^{K-1} d[m, k] \cdot e^{-j m \Omega_{1}} \cdot e^{-j k \Omega_{2}} .
$$

Suppose the desired frequency invariant response is $P(\sin \theta)$. By the substitution $\sin \theta=\left(\frac{\Omega_{1}}{\mu \Omega_{2}}\right)$, we can obtain the response $R\left(\Omega_{1}, \Omega_{2}\right)$. Sample $R\left(\Omega_{1}, \Omega_{2}\right)$ at the $\left(\Omega_{1}, \Omega_{2}\right)$ plane and then apply an inverse discrete Fourier transform (IDFT) to the resultant 2-D data, we will obtain the corresponding coefficients $d[m, k]$ with an appropriate window function to fit the spatial and temporal dimensions of the array. The precise steps can be found in $[5,6]$.

To avoid aliasing in both the spatial and temporal domains, we can choose $d_{x}=\frac{\lambda_{\min }}{2}$ and $T=\frac{c}{2 \lambda_{\min }}$, where $\lambda_{\min }$ is the wavelength corresponding to the maximum frequency of interest. As a result, we have $\mu=1$.

\subsection{Problems with off-broadside main beam design}

Based on the proposed design method in [6], we can design a frequency invariant beamformer with its main beam in an arbitrary direction. However, although the design result for a broadside main beam is very good, for the design with an offbroadside main beam, given the same number of array sensors and attached FIR coefficients, it is not as good as the broadside main beam case. Here we give two design examples for an equally spaced linear array with 21 sensors and a digital filter length of 25 . One is for a broadside main beam and one is for an off-broadside main beam. The resultant beam pattern for the broadside main beam is shown in Fig. 2, which has a very good frequency invariant property for $\Omega>0.25 \pi$.

The example for the off-broadside main beam is shown in Fig. 3, where its main beam is in the direction of $\theta=-30^{\circ}$ and the variation of the response over different frequencies is clearly visible. This problem can be explained by the con-

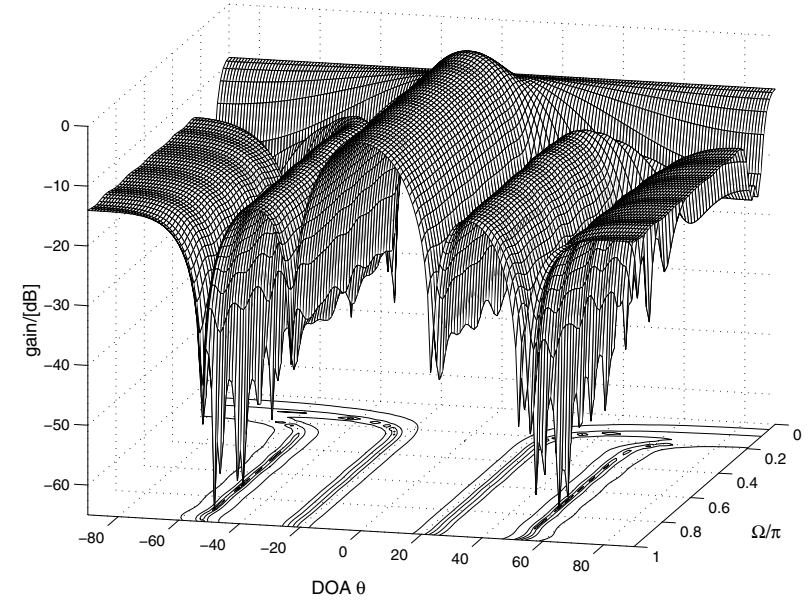

Fig. 2. The designed beam pattern for the linear array with a broadside main beam $(\mu=1)$.

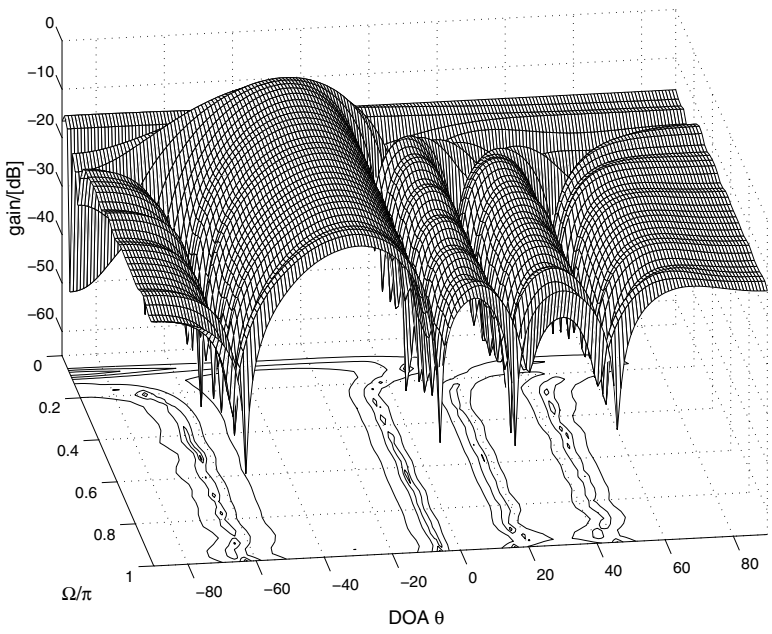

Fig. 3. The designed beam pattern of a linear array $(\mu=1)$ with its main beam at $\theta=-30^{\circ}$. 


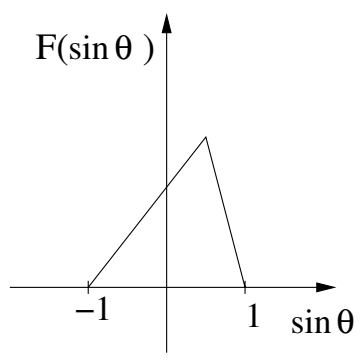

Fig. 4. A desired frequency invariant beam pattern with offbroadside main beam.

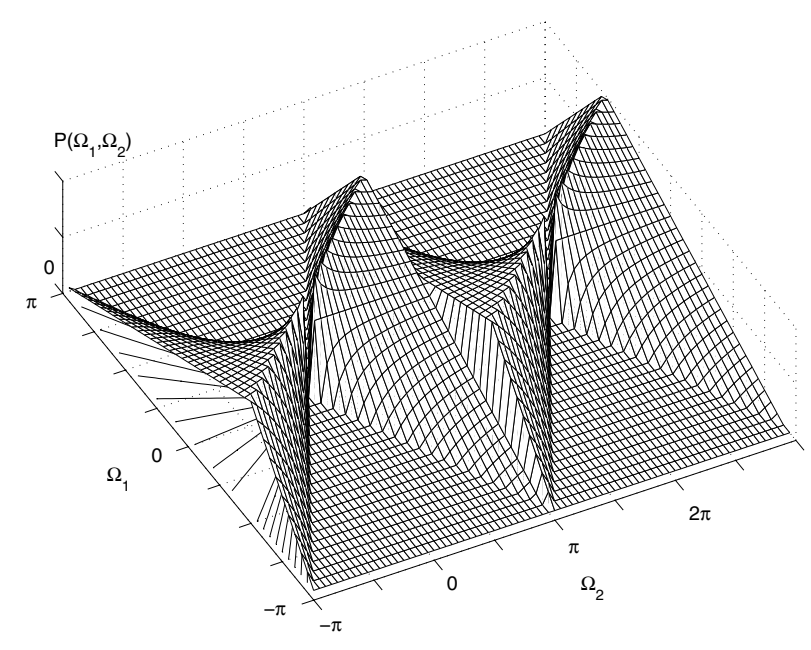

Fig. 5. The discontinuity of $P\left(\Omega_{1}, \Omega_{2}\right)=F\left(\frac{\Omega_{1}}{\Omega_{2}}\right)$ when the desired main beam is off broadside as shown in Fig. 4.

siderable discontinuity of the periodic function $P\left(\Omega_{1}, \Omega_{2}\right)$ at $\Omega_{2}=\cdots,-3 \pi,-\pi, \pi, 3 \pi, \cdots$, when the main beam is not pointing to broadside, as shown in Figs. 4 and 5. Because of the discontinuity incurred when sampling $P\left(\Omega_{1}, \Omega_{2}\right)$ and subsequently applying the inverse DFT, the response of $P\left(\Omega_{1}, \Omega_{2}\right)$ around this area cannot be controlled well. This leads to a poor performance of the proposed method, especially for frequencies close to $\pi$. This problem also occurs, although less pronounced, even if the main beam is at broadside but the beam pattern is non-symmetric with respect to it.

\section{SOLUTIONS TO THE OFF-BROADSIDE MAIN BEAM DESIGN AND EXAMPLES}

Since the problem with the off-broadside main design is due to the considerable discontinuity of the periodic function $P\left(\Omega_{1}\right.$,

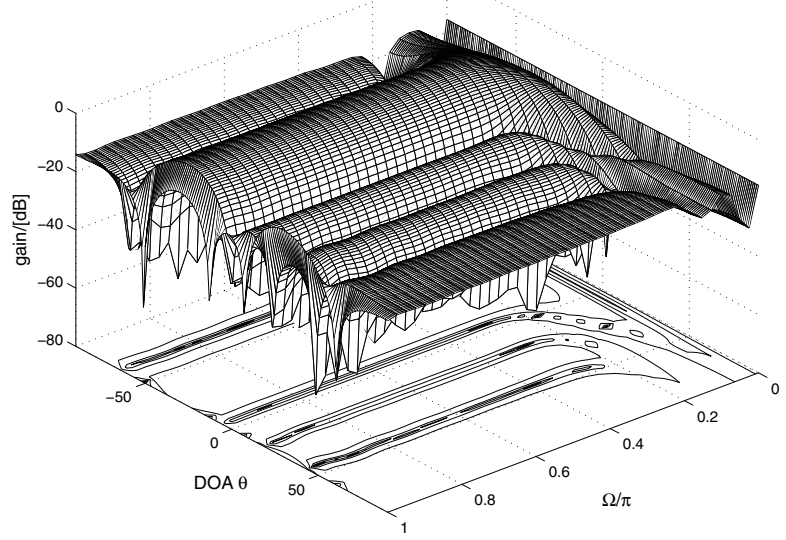

Fig. 6. The design example with an off-broadside main beam (in the direction $-30^{\circ}$ ) obtained by the first proposed method.

$\left.\Omega_{2}\right)$ at $\Omega_{2}=\cdots,-3 \pi,-\pi, \pi, 3 \pi, \cdots$, we can sample the function $P\left(\Omega_{1}, \Omega_{2}\right)$ in the $\Omega_{2}$ direction more densely and then permit more coefficients for the corresponding temporal dimension of the beamformer after truncation. Alternatively, for simplicity, we can sample $P\left(\Omega_{1}, \Omega_{2}\right)$ by a large number in both the directions of $\Omega_{1}$ and $\Omega_{2}$. After the inverse DFT, we can truncate the results with a rectangular window and leave much more coefficients in the temporal dimension (FIR filter length) than in the spatial dimension (sensor number). In the example shown in Fig. 6, we sampled $P\left(\Omega_{1}, \Omega_{2}\right)$ by $256 \times 256$ points and then truncated the IDFT results to $21 \times 127$. Comparing this result with Fig. 3, we can clearly see the significantly improved frequency invariant property. However, due to the discontinuity, no matter how many coefficients we keep for the FIR filters, its performance at $\Omega=\pi$ will never be as good as the other frequencies.

Another solution to the problem is to design a broadside main beam first, and then steer the array to the desired direction by means of appropriate delays implemented by either some analogue devices or FIR/IIR filters [1,7]. Thus, the main beam is shifted to the desired direction. As a special case, for a delay over the whole normalised frequency range $[0 \pi]$, we can use a series of truncated sinc functions. Such a design example is shown in Fig. 7, where a good frequency invariance is achieved. The problem with the frequency $\Omega=\pi$ is due to the fact that the delay can not be approximated well by the sinc function at $\Omega=\pi$. Moreover, comparing this 


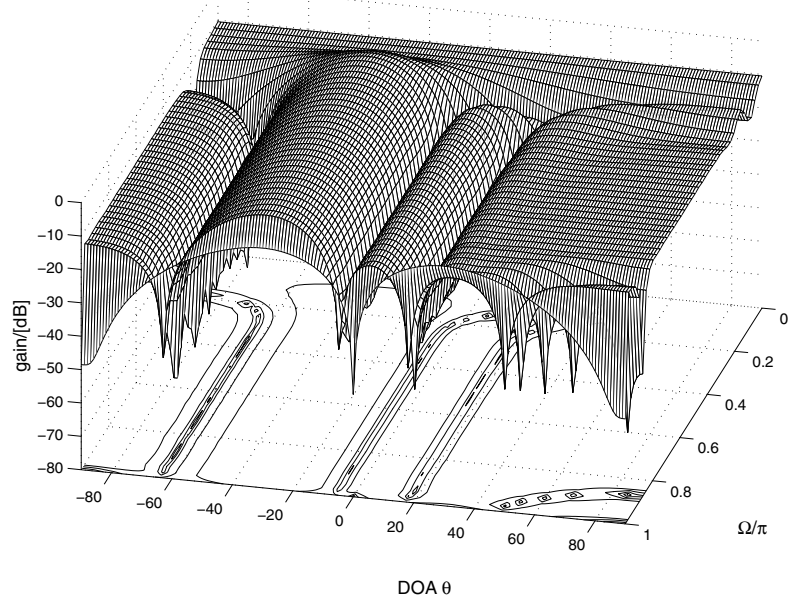

Fig. 7. The design example with an off-broadside main beam $\left(-30^{\circ}\right)$ obtained by the second proposed method.

example with the one in Fig. 6, we can also observe the big difference at the sidelobe region between about $40^{\circ}$ and $90^{\circ}$. In the next, we try to give an explanation to this difference.

Suppose the desired off-broadside main beam direction is $\theta_{0} \in[-\pi / 2 \pi / 2]$. Then after adding the appropriate steering delays for the direction $\theta_{0}$ to the broadside main beam design result, the new beam pattern can be expressed as (assume $\mu=$ 1)

$$
R(\Omega, \theta)=\sum_{m=0}^{M-1} \sum_{k=0}^{K-1} d[m, k] \cdot e^{-j m \Omega\left(\sin \theta-\sin \theta_{0}\right)} \cdot e^{-j k \Omega} .
$$

Without loss of generality, we assume $\theta_{0}<0$. Then for $-1<\sin \theta \leq\left(1+\sin \theta_{0}\right)$, we have

$$
-1<-1-\sin \theta_{0}<\sin \theta-\sin \theta_{0} \leq 1 .
$$

Then the beam response of the steered design at $\theta$ for $-1<$ $\sin \theta \leq\left(1+\sin \theta_{0}\right)$ will be the same as the response of the original broadside design at

$$
\hat{\theta}=\arcsin \left(\sin \theta-\sin \theta_{0}\right) .
$$

However, for $\left(1+\sin \theta_{0}\right)<\sin \theta \leq 1$, we have $(\sin \theta-$ $\left.\sin \theta_{0}\right)>1$. Then the shift relationship can not be expressed as (5) any more and we need to further consider the following two cases bearing in mind the periodicity of the function $e^{-j m \Omega}$ :
1. For $\Omega \leq \frac{\pi}{\sin \theta-\sin \theta_{0}}$, we have $\Omega\left(\sin \theta-\sin \theta_{0}\right) \leq \pi$, since $\left(\sin \theta-\sin \theta_{0}\right)>1$, it seems that we can not find any correspondence between the steered pattern and the original broadside pattern for this case.

2. For $\Omega>\frac{\pi}{\sin \theta-\sin \theta_{0}}$, we have

$$
\Omega\left(\sin \theta-\sin \theta_{0}\right)>\pi .
$$

Then we have

$$
e^{-j m \Omega\left(\sin \theta-\sin \theta_{0}\right)}=e^{-j m \Omega\left(\sin \theta-\sin \theta_{0}-\frac{2 \pi}{\Omega}\right)} .
$$

If $\sin \theta-\sin \theta_{0}-\frac{2 \pi}{\Omega}<-1$, i.e.

$$
\Omega<\frac{2 \pi}{1+\sin \theta-\sin \theta_{0}},
$$

then we come to the same conclusion as in the first case. Otherwise, we have

$$
\Omega \geq \frac{2 \pi}{1+\sin \theta-\sin \theta_{0}} .
$$

Then we can assume

$$
\sin \hat{\theta}=\sin \theta-\sin \theta_{0}-\frac{2 \pi}{\Omega} .
$$

Then the response of the steered design for this case will be the same as the response of the original broadside design at frequency $\Omega$ and direction of arrival angle $\hat{\theta}=\arcsin \left(\sin \theta-\sin \theta_{0}-\frac{2 \pi}{\Omega}\right)$.

Note since $\left(\sin \theta-\sin \theta_{0}\right)>1$, we have

$$
\frac{2 \pi}{1+\sin \theta-\sin \theta_{0}}>\frac{\pi}{\sin \theta-\sin \theta_{0}} .
$$

This is a complicated relationship and not as straightforward as in the narrowband case. However, there is another way to understand the relationship between the steered response and the original one.

Since $\theta_{0}<0$, we have $\left|\sin \theta-\sin \theta_{0}\right| \leq\left(1-\sin \theta_{0}\right)$ and $1-\sin \theta_{0}=\hat{\mu}>1$, then (3) can be rewritten as

$$
R(\Omega, \theta)=\sum_{m=0}^{M-1} \sum_{k=0}^{K-1} d[m, k] \cdot e^{-j m \hat{\mu} \Omega \frac{\sin \theta-\sin \theta_{0}}{\hat{\mu}}} \cdot e^{-j k \Omega} .
$$

Since $\left|\frac{\sin \theta-\sin \theta_{0}}{\hat{\mu}}\right|<1$, we assume

$$
\sin \hat{\theta}=\frac{\sin \theta-\sin \theta_{0}}{\hat{\mu}} .
$$




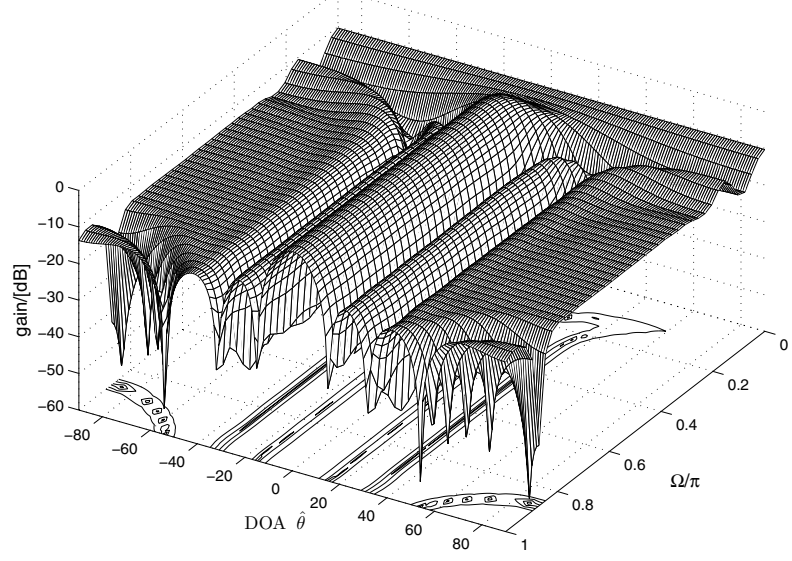

Fig. 8. The beam pattern with the inter-element spacing increased by 1.5 and the same set of coefficients as in Fig. 2.

Then (12) changes to

$$
R(\Omega, \theta)=\sum_{m=0}^{M-1} \sum_{k=0}^{K-1} d[m, k] \cdot e^{-j m \hat{\mu} \Omega \sin \hat{\theta}} \cdot e^{-j k \Omega} .
$$

Then the beam response of the steered design at $\theta \in[-\pi / 2 \pi / 2]$ will be the same as the response of the original broadside design at $\hat{\theta} \in\left[\arcsin \left(\frac{-1-\sin \theta_{0}}{\hat{\mu}}\right) \frac{\pi}{2}\right]$ with the inter-element spacing increased by $\hat{\mu}$. Now for the example of $\theta_{0}=-30^{\circ}$, we have $\hat{\mu}=1.5$ and $\arcsin \left(\frac{-1-\sin \theta_{0}}{\hat{\mu}}\right) \approx-20^{\circ}$. We can draw the response of (14) given the same set of coefficients for the example of Fig. 2. The result is shown in Fig. 8. Compared to the beam pattern of Fig. 7, we can see a clear match (although nonlinear) between Fig. 7 and that of Fig. 8 for $\hat{\theta} \in\left[-20^{\circ} 90^{\circ}\right]$.

Based on the above representative examples, the first solution is generally superior over the first one with its associated sidelobe problem. However, in the second approach the required delays can be designed independent of the specific beamformer, which may potentially provide some advantage in some applications. Moreover, the sidelobe problem may not affect the system's performance if the attenuation in these areas is high enough.

\section{CONCLUSIONS}

Two solutions to the design of frequency invariant beamformers with off-broadside main beams have been proposed. The first approach increases the temporal dimension of the beamformer in combination with a denser sampling in the Fourier domain, where a desired response is to be matched. A second one is to design a broadside main beam first, which is thereafter steered to the desired direction by a series of appropriate steering delays. Two design examples have been shown revealing the characteristics of the two methods with a detailed analysis to the effect of the steering delays in the second one.

\section{REFERENCES}

[1] D. P. Scholnik and J. O. Coleman, "Formulating wideband array-pattern optimizations," in Proc. IEEE International Conference on Phased Array Systems and Technology, Dana Point, California, May 2000, pp. 489-492.

[2] S.F. Yan and Y. L. Ma, "Design of FIR beamformer with frequency invariant patterns via jointly optimizing spatial and frequency responses," in Proc. IEEE International Conference on Acoustics, Speech, and Signal Processing, Philadelphia, USA, March 2005, pp. 789-792.

[3] H. Duan, B. P. Ng, C. M. See, and J. Fang, "Applications of the srv constraint in broadband pattern synthesis," Signal Processing, vol. 88, pp. 1035-1045, April 2008.

[4] D. B. Ward, R. A. Kennedy, and R. C. Williamson, "Theory and design of broadband sensor arrays with frequency invariant far-field beam patterns," Journal of the Acoustic Society of America, vol. 97, no. 2, pp. 1023-1034, February 1995.

[5] T. Sekiguchi and Y. Karasawa, "Wideband beamspace adaptive array utilizing FIR fan filters for multibeam forming," IEEE Transactions on Signal Processing, vol. 48, no. 1, pp. 277-284, January 2000.

[6] W. Liu, S. Weiss, J. G. McWhirter, and I. K. Proudler, "Frequency invariant beamforming for two-dimensional and three-dimensional arrays," Signal Processing, vol. 87, pp. 2535-2543, November 2007.

[7] W. S. Lu and T. B. Deng, "An improved weighted leastsquares design for variable fractional delay FIR filters," IEEE Transactions on Circuits and Systems - II: Analog and Digital Signal Processing, vol. 46, no. 8, pp. 10351040, August 1999. 